\title{
A importância dos estudos métricos da informação na construção de indicadores para a formação e desenvolvimento de coleções
}

\author{
Nadia Aurora Vanti Vitullo \\ Professora Adjunta do Dep. de Ciência da Informação da Universidade Federal do Rio Grande do \\ Norte (UFRN) \\ Doutora em Comunicação e Informação pela UFRGS \\ E-mail: nvanti@ufrnet.br \\ Raimunda Fernanda dos Santos \\ Professora substituta Departamento de Ciência da Informação da Universidade Federal do Rio \\ Grande do Norte (UFRN) \\ Mestre em Ciência da Informação pela UFPE \\ E-mail: nanda_florania@hotmail.com
}

\begin{abstract}
RESUMO:
Enfatiza a importância dos estudos métricos da informação na construção de indicadores para a formação e desenvolvimento de coleções. Objetiva abordar os métodos quantitativos como instrumentos de administração para a mensuração de dados, os quais podem facilitar o estabelecimento de diagnósticos e de avaliações a fim de otimizar a tomada de decisão para a formação e desenvolvimento de coleções nas unidades de informação. Utiliza como metodologia as pesquisas bibliográficas e eletrônicas as quais subsidiaram o desenvolvimento do estudo. Conclui destacando que a análise quantitativa da literatura, como instrumento de administração, pode acelerar o alcance da meta do bibliotecário que é a de maximizar a utilização social dos registros de informação para fins de benefício de seus usuários.
\end{abstract}

Palavras-chave: Estudos métricos da informação. Unidades de Informação. Indicadores. Formação e desenvolvimento de coleções.

\begin{abstract}
:
It emphasizes the importance of metric information studies in the construction of indicators for training and development of collections. Aims to address the quantitative methods as management tools for measurement data, which can facilitate the establishment of diagnoses and evaluations in order to optimize decision-making to the training and development of collections in information units. Used as methodology the bibliographical and electronic research which subsidized the development of the study. It concludes highlighting the quantitative analysis of the literature, as a management tool, can accelerate the achievement Librarian goal is to maximize the social use of information records for benefit purposes of its members.
\end{abstract}

Keywords: Metric studies of information. Information units. Indicators. Training and development of collections. 


\section{INTRODUÇÃO}

No hodierno contexto, a evolução tecnológica e social tem provocado diversas mudanças no que concerne à tomada de decisão nas estruturas organizacionais e na gestão de unidades de informação. Partindo desse pressuposto, a atuação do profissional da informação também tem se modificado em virtude da grande quantidade de informações, bem como da agregação de valor aos produtos e serviços de informação.

As unidades de informação têm a missão de prover a infraestrutura bibliográfica e informacional objetivando satisfazer as demandas de seus usuários . Portanto, uma das atividades que mais necessita de atenção na administração de uma unidade de informação é o desenvolvimento de coleções, uma das mais importantes atividades intelectuais do profissional da informação.

Nessa concepção, as coleções que eram formadas de maneira rudimentar exigem atualmente uma gestão criteriosa e atenta em seu processo de desenvolvimento, de modo que seja possível determinar diretrizes para nortear a sua implementação através de dados quantitativos e estudos métricos como elementos para o seu planejamento eficaz.

Dessa forma, este artigo tem como objetivo abordar os métodos quantitativos como instrumentos de administração para a mensuração de dados, os quais podem facilitar o estabelecimento de diagnósticos e de avaliações a fim de otimizar a tomada de decisão para a formação e desenvolvimento de coleções nas unidades de informação.

Esses e alguns outros aspectos serão abordados nesta pesquisa que utiliza como metodologia pesquisas bibliográficas e eletrônicas as quais subsidiaram o desenvolvimento do estudo.

\section{A FORMAÇÃO E O DESENVOLVIMENTO DE COLEÇÕES EM UNIDADES DE INFORMAÇÃO}

A preocupação com o desenvolvimento de coleções em unidades de informação encontra-se acentuada nos últimos anos. Este fenômeno é decorrente do 
significativo crescimento da quantidade de materiais informacionais e da impossibilidade de seu tratamento.

Dessa forma, desenvolver coleções é uma das funções básicas da gestão de unidades de informação haja vista que requer planejamento para a tomada decisão. Nesse entendimento, conforme Vergueiro (1993 apud WEITZEL 2002, p. 62) a expressão desenvolvimento de coleções ganhou impulso a partir da década de sessenta, quando nos Estados Unidos, "apesar dos fortes investimentos em construções de prédio para alocação das coleções, percebeu-se que não era racional adquirir tudo o que era produzido".

Assim, antigamente ocorria uma enorme preocupação em aglomerar materiais bibliográficos haja vista que os grandes acervos representavam garantia de poder e a sua disponibilidade era o foco central, desconsiderando assim o aspecto qualitativo das informações.

Destarte, de acordo com Weitzel (2002, p.61) "desde os tabletes de argila ao documento eletrônico não há como formar e desenvolver coleções sem se deparar com questões próprias da natureza do processo". Esse processo envolve desde o espaço físico para disponibilizar esse material até a busca e o fornecimento de material necessário para satisfazer as necessidades informacionais dos usuários.

Assim, o advento das tecnologias de informação e comunicação, bem como a sua adoção em larga escala pelas unidades de informação do mundo todo, trouxeram de volta indagações provenientes da natureza do processo de seleção e organização das coleções seja no ambiente tradicional ou no ambiente digital: o que selecionar? Por que e para quem?

Nessa concepção, as coleções passaram a ser produto resultante das necessidades institucionais e, consequentemente, de seus clientes internos e externos, pois o modelo de unidade de informação centrada no armazenamento para fins de preservação do conhecimento deu lugar ao novo modelo baseado no acesso às informações de interesse a partir do seu estoque ilimitado. Assim, Weitzel (2002, p.64) afirma que:

Desenvolver coleções é, portanto, uma atividade técnica comprometida com a sistematização de determinada área sob o enfoque institucional em relação aos interesses de quem mantém a biblioteca. Trata-se da construção de um pequeno núcleo temático em bases definidas e determinadas, o qual dá sentido ao que está disperso no mundo caótico das 
informações. Em outras palavras, desenvolvimento de coleções é uma disciplina que procura organizar o conhecimento registrado sob enfoques e filtros específicos - uma solução técnica desencadeada pela explosão bibliográfica.

A partir das conceituações da autora supracitada, depreende-se que a atividade de desenvolver coleções tornou-se imprescindível para se administrar acervos nas unidades de informação, levando em consideração que a mesma funciona como um filtro do conhecimento registrado para o consumo adequado por parte dos seus usuários.

Mediante tais considerações, equacionar recursos (financeiros, materiais e humanos) e subsidiar o bibliotecário na intermediação acervo-usuário a fim de promover o compartilhamento de informações de qualidade são algumas funções da atividade de formação e desenvolvimento de coleções.

Sendo assim, no hodierno contexto informacional, o diferencial da formação e desenvolvimento de coleções se dá através da seleção adequada das informações por meio da obediência a padrões que garantam a sua confiabilidade e a disponibilidade de obras de qualidade em diversos suportes informacionais (tais como livros impressos e digitais, periódicos, etc.). Com isso, as coleções precisam crescer coerentemente em todas as áreas evitando que o acervo cresça de maneira desordenada e desproporcional sem objetivos ou metas concretas.

Nessa perspectiva, a adoção das tecnologias de informação e comunicação em unidades de informação inovou todos os processos e práticas biblioteconômicas nesse contexto. É nesse momento que podemos perceber a importância do bibliotecário em funções gerenciais, uma vez que além de outras atividades este profissional tem a responsabilidade de oferecer material informacional suficiente, adequado e organizado para tentar suprir as necessidades daqueles que buscam informação.

Nesse sentido, no que concerne às funções gerenciais Barbalho e Beraquet (1995 apud BRANÍCIO; CASTRO FILHO, 2007, p.146) enfatizam que:

As atividades gerenciais são aquelas que se relacionam às atividades que mantém as unidades funcionando de forma satisfatória, a saber: o planejamento, relacionado ao estabelecimento de metas e formas de atingilas; a organização, voltada para as decisões sobre divisão do trabalho; o desempenho, relacionado à execução do trabalho em si; o controle, voltado para assegurar o alinhamento entre o trabalho que está sendo executado e 
o planejado; e a revisão, voltada para a análise e para o resumo do que foi feito.

Portanto, em relação à atividade gerencial de desenvolvimento de coleções é importante que o profissional da informação interprete a missão da instituição a qual o mesmo faz parte e elabore um plano ou política de desenvolvimento de coleção que descreva os objetivos, a curto e longo prazo, da biblioteca para suas coleções, levando-as em consideração e correlacionando-as com aspectos burocráticos (planos fiscais, orçamentos dos projetos etc.) e fatores do ambiente, como a demanda do usuário, suas necessidades e expectativas, bem como a história das coleções.

Para dar segmento a essa consideração, Maciel (2000) aponta algumas decisões que devem ser tomadas nas políticas que conduzirão o processo de formação e desenvolvimento de coleção de unidades de informação. Tais decisões serão elencadas no quadro a seguir:

\section{Quadro 1- Decisões a serem tomadas em políticas de formação e desenvolvimento de coleções}

*Determinação das áreas que irão compor o acervo da unidade de informação

*Indicação do tipo de material que irá compor o acervo, independente do seu suporte físico

*Estabelecimento de diretrizes para a avaliação das coleções podendo designar a periodicidade com que estas serão analisadas.

*Elaboração de critérios e prioridades que orientarão o processo de formação e desenvolvimento das coleções (incluindo as etapas de seleção, aquisição, desbaste, etc.)

*Definição da quantidade de exemplares por título

*Formação de diretrizes para a preservação e conservação do acervo no que concerne às condições ambientais ideais para os tipos de documentos ali presentes.

*Determinação de prazos para a revisão e avaliação das políticas.

Fonte: Elaborado pelas autoras.

Assim, torna-se perceptível que o processo de adquirir coleções deve ser submetido a diversos procedimentos formais os quais refletem sobre o papel do administrador de bibliotecas no âmbito dos serviços de informação. Nesse sentido, o bibliotecário responsável pelo setor de formação e desenvolvimento de coleções de Rev. Inf. na Soc. Contemp., Natal, RN, v.1, n.2, jan./jun., 2017| 
uma unidade de informação deve ampliar produtos visando à satisfação das necessidades de seus usuários ocasionando assim mudanças que melhorem o desempenho dos serviços.

Diante disso, para que a seleção de materiais aconteça de maneira mais satisfatória é preciso- além de conhecer o seu usuário- se preocupar com alguns critérios, os quais serão apontados no quadro abaixo de acordo com as idéias de Vergueiro (1997), sendo eles:

Quadro 2- Critérios de seleção de materiais

\begin{tabular}{c} 
Autoridade \\
Precisão \\
Imparcialidade \\
Atualidade \\
\hline Cobertura e tratamento \\
Conveniência \\
\hline Relevância/Interesse \\
Custo \\
\hline
\end{tabular}

Fonte: Elaborado pelas autoras.

No critério de autoridade o autor supracitado aponta que a qualidade do material está relacionada com o autor, editor, ou patrocinador, no entanto não existem garantias a respeito deste critério. A precisão evidencia o quanto a informação de um documento é exata, rigorosa e correta. Em relação à imparcialidade é necessário verificar se os assuntos dos documentos são apresentados sem favoritismo a algum ponto de vista, assim este critério poderá ou não ser pré-requisito para aquisição do item. A atualidade é um critério decisivo em bibliotecas que valorizam dados atuais. Estes critérios irão variar de acordo com a área, as mudanças políticas e estruturais na sociedade, nesse sentido o profissional deve manter-se atento à veracidade destas atualidades. 
A cobertura e/ou tratamento diz respeito à abordagem dos assuntos e seus aspectos. A determinação deste critério é dada pela especificidade da clientela e/ou coleção, nesse aspecto visa verificar se todos os detalhes do assunto foram contemplados e se foram abordados todos os aspectos importantes. No que concerne ao critério de conveniência é necessário verificar o vocabulário e o visual do trabalho, os quais serão determinados pela idade e desenvolvimento intelectual dos usuários e no critério de relevância/ interesse é observado se o documento é relevante para a experiência do usuário e em relação ao custo é necessário buscar alternativas financeiras mais compensadoras para a unidade de informação.

Nesse contexto podemos enfatizar a importância do profissional da informação conhecer quais os materiais estão presentes no acervo da unidade de informação e o que é necessário ser adquirido, levando em consideração a realidade da instituição (fatores políticos, econômicos culturais e sociais) em que a mesma está inserida.

Além disso, Vergueiro (1995, p.9) menciona que "O bibliotecário deverá sempre participar com seu conhecimento de coleção, propondo uma direção coerente para o acervo e garantindo, assim que os objetivos para ela estabelecidos não se percam com o passar do tempo". Por essa razão, este profissional deve se atentar a alguns requisitos básicos para suprir as necessidades de informação dos usuários, tais como:

${ }^{*}$ Conhecer bem a comunidade em que a unidade de informação está inserida;

*Saber julgar o valor dos materiais informacionais;

${ }^{*}$ Conhecer a realidade financeira da instituição e estar a par do orçamento disponível para o desenvolvimento de coleções;

*Possuir experiências profissionais na área de aquisição, seleção, desbaste e descarte de documentos;

Com isso em mente, para que ocorra uma gestão de qualidade nas unidades de informação é importante que o bibliotecário tenha conhecimento de uma visão geral dos processos e do local onde o mesmo atua. Este profissional deve conhecer os usuários (reais e potenciais) e os serviços ofertados, entre outros aspectos, com o objetivo de fazer o planejamento de acordo com as necessidades da instituição onde o mesmo atua. 
Nesse sentido, o bibliotecário responsável pela atividade de formação e desenvolvimento de coleções deve possuir diversas competências que envolvem ações relacionadas com os usuários, acervos, recursos tecnológicos, financeiros e com a prática organizacional.

Sob esse viés, a seguir serão apontados alguns fatores importantes que este profissional deve levar em consideração enquanto gestor de unidades de informação no setor de desenvolvimento de coleções:

${ }^{*}$ Conhecer o usuário $\rightarrow$ identificar e interpretar as necessidades e demandas de informação. Marchiori (2002, p.77) afirma que "De maneira geral, o gestor da informação vai mapear os pontos de uso de informação identificando as necessidades e requisitos indicados/negociados junto a seus clientes".

*Avaliar fontes de informação $\rightarrow$ reconhecer e identificar tanto quantitativa como qualitativamente as fontes de informação da área em que a organização atua. É importante também selecionar e listar as bases de dados confiáveis sobre as áreas de conhecimento que interessam à unidade de informação.

*Selecionar e tratar tecnicamente a informação $\rightarrow$ localizar e agregar fontes para a construção da informação envolvendo atividades de filtragem, organização e análise com o intuito de agregar valor a produtos e serviços.

*Gerenciar a informação $\rightarrow$ gerenciar dados a fim de analisá-los para serem oportunamente disponibilizados ao usuário como informação e como subsídio para a produção do conhecimento.

*Mediar a informação $\rightarrow$ intermediar as demandas da unidade de informação e o universo de informações acessíveis sendo agente mediador da informação, auxiliando na sua busca e seleção.

*Buscar e gerar informações estratégicas $\rightarrow$ oferecer informações de qualidade e na íntegra, atualizadas e precisas, de relevância e valor agregado para a tomada de decisão.

*Disseminar seletivamente a informação $\rightarrow$ divulgar e gerir os fluxos de informação, distribuindo-a em sua forma original ou como produtos elaborados a partir dela.

Em linhas gerais, como é possível perceber, a atividade de formação e desenvolvimento de coleções não se trata apenas de um simples processo técnico ou momento determinante da construção de coleções nas práticas Rev. Inf. na Soc. Contemp., Natal, RN, v.1, n.2, jan./jun., 2017| 
biblioteconômicas. Esta atividade reflete na execução do papel social atribuído ao bibliotecário que possui a responsabilidade de gerenciar coleções para e com o público a fim de satisfazer as suas necessidades informacionais.

Destarte, o levantamento de informações para tomada de decisão no setor de formação e desenvolvimento de coleções pode ser também realizado através de estudos métricos da informação- os quais serão mencionados no tópico a seguir.

\section{OS ESTUDOS MÉTRICOS DA INFORMAÇÃO}

Desde que a ciência passou a ser determinante para o crescimento econômico e social para toda nação, nasce o interesse em coletar informações seja em qual for a atividade de Ciência e Tecnologia, para que estas estejam planejadas, monitoradas e avaliadas.

Segundo Mugnaini; Carvalho; Campanatti-Ostiz (2006, p.316) "para se entender a evolução da ciência, como forma de expressão do conhecimento humano produzido são utilizadas técnicas de medição", aplicadas em estudos métricos os quais são realizados para a composição ou fortalecimento de indicadores que possibilitam traçar um perfil do mundo científico em âmbito nacional e internacional.

Atualmente, os principais métodos e técnicas para a avaliação quantitativa da ciência são utilizados nos estudos métricos da informação, com várias aproximações (teóricas e metodológicas) e diferentes denominações em função de seus objetivos a serem atingidos e objetos de estudo a serem pesquisados. Sob esse viés, segundo Noronha e Maricato (2008, p.118) "quaisquer que sejam os métodos empregados nos estudos métricos da ciência, há que se considerar um conjunto de variáveis componentes do processo das atividades científicas, que podem e devem ser medidas, para que as investigações realizadas atinjam resultados positivos".

Nesse sentido, de acordo com Lima (1986) o desenvolvimento científico e tecnológico intensificou o uso de métodos matemáticos, em processos acelerados pelo uso do computador e se constituindo em importante desenvolvimento na história da ciência.

De outro modo disposto, Morales (1985, grifo nosso) relaciona o resultado da aplicação de métodos quantitativos e a área de conhecimento em que os mesmos Rev. Inf. na Soc. Contemp., Natal, RN, v.1, n.2, jan./jun., 2017| 
estão interligados, como: bibliometria com biblioteconomia, cientometria com ciência da ciência e informetria com ciência da informação.

A Bibliometria estuda os aspectos quantitativos da produção, disseminação e uso da informação registrada. Ela desenvolve padrões e modelos matemáticos para medir esses processos, usando os resultados para elaborar previsões e apoiar tomadas de decisões (TAGUE- SUTCLIFFE, 1992). Nesse segmento, os estudos bibliométricos vêm se tornando cada vez mais atraentes devido os novos recursos tecnológicos disponíveis- os quais facilitaram a coleta de dados nas fontes primárias, bem como o seu tratamento.

Com a ampliação do interesse por esses estudos, surgem novas técnicas e métodos como sub-campos da Bibliometria como a Cientometria, a Informetria e entre outros que se assemelham por serem métodos quantitativos e se distinguem quanto aos seus objetos de estudo.

Destarte, de acordo com o autor supracitado a Cientometria (também denominada Cienciometria) é o estudo dos aspectos quantitativos da ciência como disciplina ou atividade econômica, ou seja, é um segmento da sociologia da ciência, sendo aplicada no desenvolvimento de políticas científicas.

Em nítido contraste, a Informetria é conceituada na literatura como o estudo dos aspectos quantitativos da informação em qualquer formato, e não apenas registros catalográficos ou bibliografias, podendo incorporar, utilizar e ampliar outros estudos de avaliação da informação que estão fora dos limites tanto da Bibliometria como da Cientometria (embora estes dois sejam os estudos métricos mais difundidos).

Para dar segmento a essa consideração, o quadro a seguir aponta os métodos e técnicas bibliométricas existentes, bem como descreve as suas finalidades e os seus respectivos objetos de estudo. 
Quadro 3- Métodos e técnicas bibliométricas

\begin{tabular}{|c|c|c|}
\hline Técnica & Finalidade & Objetos de estudo \\
\hline BIBLIOMETRIA & $\begin{array}{l}\text { Produção e uso de documentos } \\
\text { Organização de serviços bibliográficos. }\end{array}$ & $\begin{array}{l}\text { Documentos (livros, } \\
\text { artigos, teses...), autores, } \\
\text { usuários }\end{array}$ \\
\hline CIENCIOMETRIA & $\begin{array}{l}\text { Organização da ciência Fatores que } \\
\text { diferenciam as sub-disciplinas } \\
\text { Identificar domínios de interesse }\end{array}$ & $\begin{array}{ll}\begin{array}{l}\text { Disciplinas, } \\
\text { áreas, }\end{array} & \text { campos, } \\
\text { especificos } & \\
\end{array}$ \\
\hline INFORMETRIA & $\begin{array}{l}\text { Medição de sistemas de informação. } \\
\text { Recuperação da informação } \\
\text { Estudo conteúdos informativos }\end{array}$ & $\begin{array}{l}\text { Palavras, documentos, } \\
\text { bases de dados }\end{array}$ \\
\hline BIBLIOTECOMETRIA & $\begin{array}{l}\text { Organização de bibliotecas } \\
\text { Administração de serviços de bibliotecas }\end{array}$ & Bibliotecas \\
\hline WEBMETRIA & Organização e uso de sites & $\begin{array}{l}\text { Páginas na internet, } \\
\text { hospedeiros }\end{array}$ \\
\hline PATENTOMETRIA & $\begin{array}{l}\text { Conhecer atividades tecnológica e } \\
\text { inovadora de países, áreas e instituições }\end{array}$ & Patentes \\
\hline
\end{tabular}

Fonte: Noronha e Maricato (2008)

Outrossim, para complementar o que é exposto no quadro acima, Oliveira e Grácio (2011, p.19) afirmam que:

\begin{abstract}
Os "Estudos Métricos" compreendem o conjunto de estudos relacionados à avaliação da informação produzida, mais especialmente científica, em diferentes suportes, baseados em recursos quantitativos como ferramentas de análise. Fundamentados na sociologia da ciência, na ciência da informação, matemática, estatística e computação, são estudos de natureza teórico-conceitual, quando contribuem para o avanço do conhecimento da própria temática, propondo novos conceitos e indicadores, bem como reflexões e análises relativas à área. São, também, de natureza metodológica, quando se propõem a dar sustentação aos trabalhos de caráter teórico da área onde estão aplicados.
\end{abstract}

Além disso, Noronha e Maricato (2008, p.116) enfatizam que "os estudos métricos da informação, sobretudo os estudos baseados na Bibliometria e na Cientometria, atuam em diferentes áreas e com temas relativamente bem definidos e aceitos pela comunidade". Dessa forma, alguns dos principais indicadores extraídos desses estudos, os quais possuem importância para a formação e desenvolvimento de coleções em unidades de informação, podem ser apresentados de modo genérico, incluindo:

- Comportamentos de uso e crescimento do acervo em bibliotecas; 
- Características e funções de diversos tipos documentais (literatura branca e cinzenta);

- $\quad$ Evolução quantitativa e qualitativa da literatura;

- $\quad$ Características de freqüência de ocorrência de palavras em textos.

- Ranking de publicações, autores, instituições, países etc.;

- $\quad$ Estudos de citação, fator de impacto;

- Relações interdisciplinares, intradisciplinares e multidisciplinares na ciência;

- $\quad$ Evolução de disciplinas, sub-disciplinas e novos conceitos;

- $\quad$ Obsolescência da informação e dos paradigmas científicos.

Portanto, quaisquer que sejam as técnicas empregadas nos estudos métricos é necessário considerar um conjunto de variáveis componentes do processo das atividades científicas, que podem e devem ser medidas, para que as investigações realizadas possam atingir resultados positivos no âmbito das unidades de informação.

\section{DADOS QUANTITATIVOS E ESTUDOS MÉTRICOS : ELEMENTOS PARA O PLANEJAMENTO EFICAZ NA FORMAÇÃO E DESENVOLVIMENTO DE COLEÇÃO}

Atualmente muitos bibliotecários não se preocupam em registrar dados estatísticos pertinentes, ou não se dão ao trabalho de analisá-los e interpretá-los. São encontradas, constantemente, em unidades de informação, caixas e caixas de formulários preenchidos pelos usuários sem que os dados tenham sido tabulados e analisados, ou até mesmo utilizados para planejamento. Com isso, o processo de coleta de dados acaba reduzido a desperdício de tempo, material e espaço.

Sendo assim, planejamento eficaz e uma política de formação e desenvolvimento de coleções em unidades de informação dependem da qualidade e quantidade de dados disponíveis e confiáveis. É indispensável, portanto, que as unidades de informação planejem adequadamente a estrutura de dados que desejam monitorar, de forma a poder dispor desses dados para o planejamento e a 
tomada de decisões fundamentadas visando também a otimização da formação e desenvolvimento de coleções.

Entretanto, segundo Almeida (2005) a análise de relatórios de diferentes bibliotecas tem mostrado que as estatísticas nem sempre servem aos propósitos do planejamento, pois, em geral, não passam de simples contagem de ocorrências ou materiais, que não resultam de estudos racionais, e são utilizadas apenas para prestar contas aos superiores.

Portanto, é necessário levar em consideração que números são importantes, mas devem estar organizados para fazer sentido e serem carregados de significado. Esta atividade pode ser desenvolvida através da escolha adequada do que será mensurado, pelo uso de quadros comparativos e ilustrativos, bem como pelo cruzamento de dados e por comentários sobre esses dados.

De acordo com Almeida (2005) os conceitos de mensuração e avaliação são muito próximos. A mensuração é o processo de atribuir números para descrever ou representar algum objeto ou fenômeno de modo padronizado, podendo levar à avaliação. Nessa concepção, a avaliação também inclui a mensuração e acrescenta componentes relacionados à pesquisa, ao planejamento e à implementação de estratégias para desenvolver as coleções.

Ainda de acordo a autora supracitada, a mensuração da eficácia pode apresentar alguns problemas, dentre eles o nível de desempenho que se pretende atingir. Além disso, muitas vezes, há registros quantitativos de resultados, como por exemplo, a quantidade de obras emprestadas, mas faltam dados qualitativos relativos ao grau de satisfação dos usuários que pediram emprestado esses materiais.

Assim, os padrões servem para orientar a definição de medidas de desempenho, que são um meio para determinar em que grau os objetivos da unidade de informação são cumpridos, os serviços executados e os materiais adequados colocados á disposição do usuário.

Whitehall (1994 apud ALMEIDA, 2005) trabalha com o conceito de medidas de desempenho. Medidas tais como o índice de penetração (quantidade de usuários ou uso do material informacional), de cobertura do acervo (proporção de demandas atendidas pelas diferentes coleções) e medidas de relevância do acervo (uso médio 
anual do acervo) podem apresentar a qualidade dos serviços da unidade de informação.

No que concerne aos estudos dos usuários das unidades de informação, através de uma abordagem quantitativa da informação coletada é possível colher dados, descrever perfis e, consequentemente, identificar as necessidades de informação dos mesmos para desenvolver coleções. Dessa forma, os indicadores quantitativos através de estudos métricos transformam os objetivos e resultados em parâmetros concretos, passíveis de verificação, os quais são representados por variáveis numéricas.

Sob esse viés, os indicadores quantitativos podem ser um número absoluto (por exemplo, número de usuários cadastrados em uma determinada unidade de informação) ou um número relativo (por exemplo, proporção de alunos de pósgraduação cadastrados em uma biblioteca universitária em relação ao número total de alunos de pós-graduação da unidade).

Entretanto, é importante enfatizar que dados numéricos, ainda que coletados sistemática e periodicamente, somente serão transformados em informação quando puderem ser analisados comparativamente e possibilitarem uma avaliação que dê subsídios a uma ação efetiva através do auxílio de alguns instrumentos.

Destarte, um instrumento para o bibliotecário analisar e avaliar as coleções e o desempenho da biblioteca é o relatório. Nessa perspectiva, o quadro de acervo deve ser montado de tal forma que permita, a qualquer momento, a obtenção de dados acumulados e atualizados para relatórios anuais ou parciais, conforme será ilustrado no quadro abaixo: 
Quadro 4 - Dados relativos ao processamento do Acervo

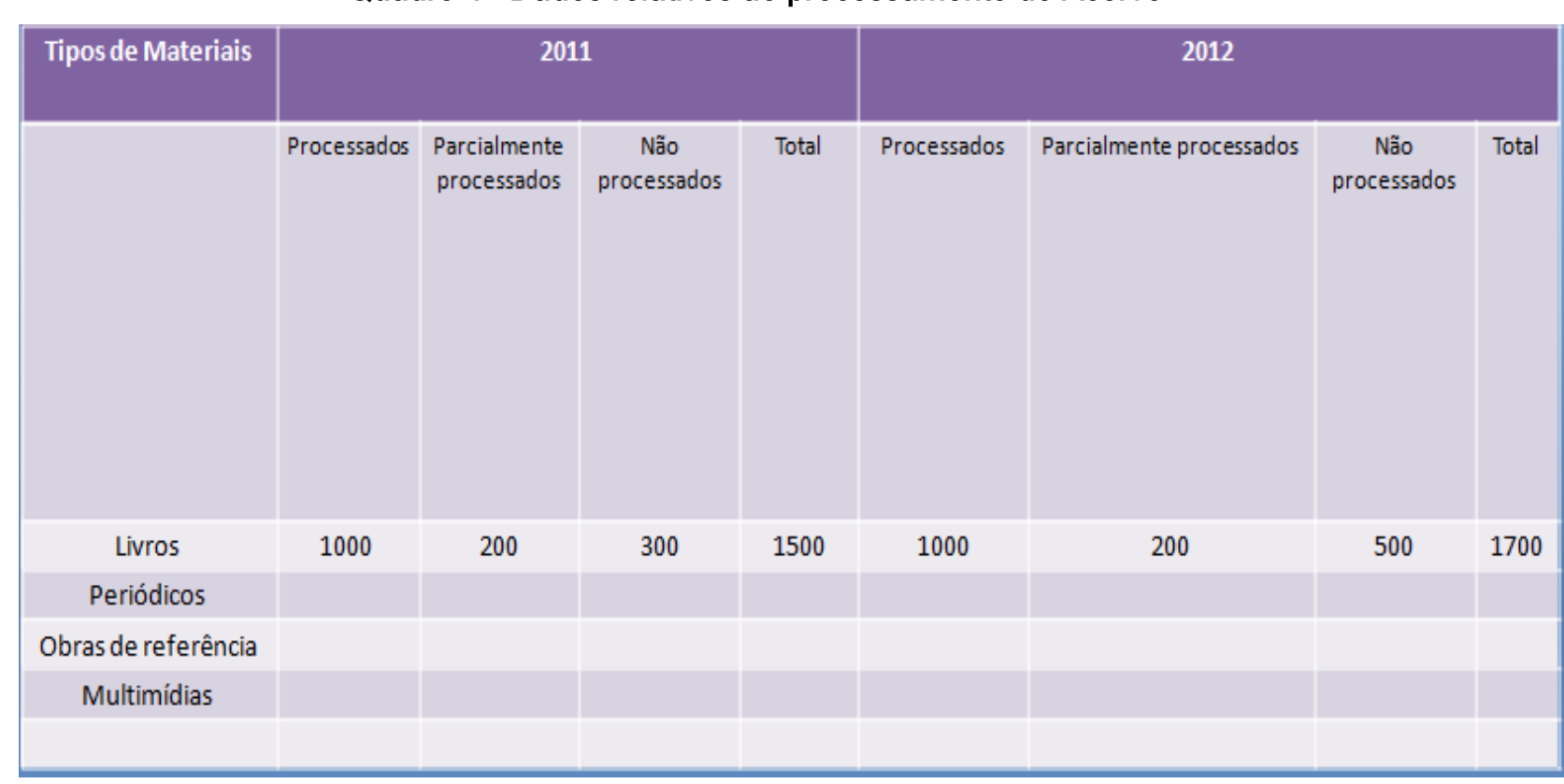

Fonte: Elaborado pelas autoras.

Diante disso é perceptível que a otimização da relação custo-benefício nas unidades de informação pode ser alcançada quando se busca a ordenação do caos documentário e a racionalização das ações através de resultados obtidos pela aplicação de métodos quantitativos e elaboração de relatórios.

\section{CONSIDERAÇÕES FINAIS}

Diante do exposto é necessário enfatizar que as técnicas e metodologias da área de desenvolvimento de coleções têm apresentado soluções para administrar conflitos entre demandas e necessidades, bem como restrições de recursos em nível local.

Sendo assim, a área de desenvolvimento de coleções vem, ao longo de sua história, apresentando soluções locais para lidar com a dispersão do conhecimento, onde cada grupo social delimita sua ação dentro do campo científico específico e suas coleções representam as características institucionais e legítimas desse grupo.

Nesse sentido, a análise quantitativa da literatura, como instrumento de administração, pode acelerar o alcance da meta do bibliotecário que é a de maximizar a utilização social dos registros de informação para fins de benefício de seus usuários. 
Entretanto, conforme enfatizado nesta pesquisa, embora existam muitos métodos quantitativos para avaliar coleções, tais métodos não devem ser o único indicador para a tomada de decisão. Faz-se necessário a combinação de métodos quantitativos e qualitativos para reunir conhecimentos técnicos e bom senso e assim estar bem embasado para a tomada de decisão.Em linhas gerais, é necessário enfatizar que, acima de tudo, o bibliotecário deve contribuir para que os recursos de informação gerem eficácia e eficiência no processo de tomada de decisões, principalmente na satisfação das necessidades dos usuários. O ponto de partida para o cumprimento das funções básicas da formação e do desenvolvimento de coleções é o planejamento. Planeja-se e projeta-se não só o próprio negócio, como também as operações e atividades que estão sendo realizadas pela unidade de informação por meio do desenvolvimento de ferramentas, processos e planos de trabalho.

Assim, a evolução dos estudos teóricos neste campo, é de extrema importância haja vista que proporciona o advento de novas abordagens no que concerne à interpretação de dados coletados, às metodologias e às variáveis possíveis de serem analisadas pelos bibliotecários no âmbito das unidades de informação.

Portanto, este artigo é de grande importância para a comunidade de pesquisadores e profissionais da informação tendo em vista que as diversas atividades de desenvolvimento de coleções sempre foram motivo de interesse dos mesmos, levando em consideração que a aplicação de estudos métricos da informação em suas práticas consiste em um diferencial para a tomada de decisão no hodierno contexto.

\section{REFERÊNCIAS}

ALMEIDA, Maria Cristina Barbosa de. Planejamento de bibliotecas e serviços de informação. Brasilia, DF: Briquet de Lemos, 2005.

ANDRADE, Diva ; VERGUEIRO, Waldomiro de Castro Santos. Aquisição de matérias de informação. Brasília: Brinquet de Lemos, 1996. 
BRANÍCIO, Simone de A. R.; CASTRO FILHO, Cláudio M. O trabalho do dirigente de unidades de informação sob diferentes perspectivas administrativas. Perspectivas em Ciência da Informação, Minas Gerais, v.12, n.3, p.142-155, set./dez. 2007.

LIMA, Regina Célia Montenegro de. Bibliometria : análise quantitativa da literatura como instrumento de administração em sistemas de informação. Ci. Inf., Brasília, v. 15, n. 2, p.127-133, jul./dez. 1986.

MACIEL, A. C.; MENDONÇA, M. A. R. Bibliotecas como organizações. Rio de Janeiro: Interciência, 2000.

MARCHIORI, P. Z. A ciência e a gestão da informação: compatibilidade no espaço profissional. Ciência da Informação, Brasília, DF, v. 31, n. 2, p. 72-79, maio/ago. 2002. Disponível em: <http://www.ibict.br/cionline/>. Acesso em: 23 maio. 2014.

MORALES; Melvyn. Informetrics and its importance. Internation Fórum on Information and Documentation, v.10, n.2, p.15-21, April 1985.

MUGNAINI, Rogério; CARVALHO, Telma; CAMPANATTI-OSTIZ, Heliane. Indicadores de produção científica: uma discussão conceitual. In: POBLACION, Dinah Aguiar; WITTER, Geraldina Porto; SILVA, José Fernando Modesto da. Comunicação \& produção científica: contexto, indicadores e avaliação. São Paulo: Angellara, 2006. p.313-340.

NORONHA, Dayse Pires; MARICATO, João de Melo. Estudos Métricos da Informação : primeiras aproximações. Enc. Bibli: Rev. Eletr. Bibliotecon. Ci. Inf., Florianópolis, n. esp., 2008.

OLIVEIRA, Ely Francina Tannuri de.; GRACIO, Maria Cláudia Cabrini. Perspectivas em Ciência da Informação, Minas Gerais, v.16, n. 4, p. 16-28, out./dez. 2011.

Disponível em: < http://www.scielo.br/pdf/pci/v16n4/v16n4a03.pdf>. Acesso em: 20 out. 2014.

TAGUE-SUTCLIFFE, J. Introduction to informetrics. Information Processing and Management, v. 28, n.1 p. 1-3, 1992.

VERGUEIRO, Waldomiro de Castro Santos. Seleção de materiais de informação. Brasília: Briquet de Lemos, 1995.

Desenvolvimento de coleções: uma nova visão para o planejamento de recursos informacionais. Ci. Inf., Brasília, v. 22, n.1, 1993. Disponível em: <http://revista.ibict.br/index.php/ciinf/article/view/1208/849> . Acesso em: 10 dez. 2014.

Seleção de materiais de informação. Brasília: Brinquet de Lemos,1997.

WEITZEL, Simone R. O desenvolvimento de coleções e a organização do conhecimento : suas origens e desafios. Perspect. Cienc. Inf., Belo Horizonte, v.7, n.1, p.61-67, jan./jun. 2002. 
Rev. Inf. na Soc. Contemp., Natal, RN, v.1, n.2, jan./jun., 2017| 